\title{
SINGULARIDAD DE SOLUCIONES PARA UN SISTEMA DE KIRCHHOFF NO LINEAL VISCOELÁSTICO CON TÉRMINO DISIPATIVO Teófanes Quispe Méndez*
}

Resumen: En el presente trabajo, estudiamos la singularidad en tiempo finito de las soluciones de un problema mixto relativo a un sistema de ecuaciones de Kirchhoff no lineal viscoelástico con término disipativo.

Palabras clave: Singularidad de soluciones, Solución Local, Sistema de ecuaciones de Kirchhoff no lineal viscoelástico, Sistema de ecuaciones integro-diferencial.

\section{BLOW-UP OF SOLUTIONS FOR A VISCOELASTIC NONLINEAR KIRCHHOFF'S SYSTEM WITH DISSIPATIVE TERM}

\begin{abstract}
In present work, we study the blow-up infinite time of solutions to the mixed problem relative to a system of viscoelastic nonlinear Kirchhoff's equations with dissipative term.

Key words: Blow-up of solutions, Local solution, System of viscoelastic nonlinear Kirchhoff's equations, System of integro-differential equations.
\end{abstract}

\section{INTRODUCCIÓN}

En este artículo consideramos el problema de valor inicial y de frontera para el siguiente sistema de ecuaciones de Kirchhoff con memoria:

$$
\begin{aligned}
u^{\prime \prime}-M\left(|\nabla u|_{2}^{2}\right) \Delta u+g_{1} * \Delta u-\Delta u^{\prime} & \left.=f_{1}(u, v) \text { en } \Omega \times\right] 0, \infty[, 1.1 \\
v^{\prime \prime}-M\left(|\nabla v|_{2}^{2}\right) \Delta v+g_{2} * \Delta v-\Delta v^{\prime} & \left.=f_{2}(u, v) \text { en } \Omega \times\right] 0, \infty[, 1.2
\end{aligned}
$$

con condiciones iniciales,

$$
\begin{aligned}
& u(x, 0)=u_{0}(x), u^{\prime}(x, 0)=u_{1}(x), \text { en } \Omega, 1.3 \\
& v(x, 0)=v_{0}(x), v^{\prime}(x, 0)=v_{1}(x), \text { en } \Omega, 1.4
\end{aligned}
$$

y condiciones de frontera,

$$
\begin{aligned}
& u(x, t)=0, \text { en } \partial \Omega \times] 0, \infty[.1 .5 \\
& v(x, t)=0, \text { en } \partial \Omega \times] 0, \infty[.1 .6
\end{aligned}
$$

donde $\Omega$ es un conjunto abierto y acotado de $\mathbb{R}^{n}$ : con frontera suficientemente regular $\partial \Omega, \nabla$ es el operador gradiente, $\Delta$ es el operador laplaciano, $M(s)$ es una función real positiva de clase $C^{1}$ para $s \geq 0, g_{i}(t)$ : $i=1,2$, son funciones reales no negativas de clase $C^{1}$ para $t \geq 0, f_{i}(s, r), i=1,2$, son funciones reales no lineales continuamente diferenciables para $(s, r) \in \mathbb{R}^{2} ; w^{\prime}:=\frac{\partial w}{\partial t} ; w^{\prime \prime}:=\frac{\partial^{2} w}{\partial t^{2}},|\nabla w|_{2}^{2}:=\int_{\Omega 2}|\nabla w(x, t)|^{2} d x \mathrm{y}$ $(g * w)(x, t):=\int_{0}^{t} g(t-s) w(x, s) d s$.

El caso $n=1$ y $u=v$, la ecuación (1.1) describe vibraciones transversales no lineales de una cuerda de material viscoelástico, fuertemente tensa entre dos puntos fijos $x=0$ y $x=L$, en cl cje $x$ del plano $x u$. En estas condiciones la ecuación resultante es

$$
\rho h u^{\prime \prime}-\left(p_{o}+\frac{E h}{2 L}\left|\frac{\partial u}{\partial x}\right|_{2}^{2}\right) \frac{\partial^{2} u}{\partial x^{2}}+g * \frac{\partial^{2} u}{\partial x^{2}}-\beta \frac{\partial^{2} u^{\prime}}{\partial x^{2}}=f(u),
$$

\footnotetext{
*UNMSMS, Facultad de Ciencias Matemáticas, e-mail: tquispem@unmsm.edu.pe
} 
donde $u=u(x, t)$ es el desplazamiento transversal en el espacio de coordenada $x$ y en el tiempo $t, \rho$ es la densidad de masa, $h$ es el área de la sección transversal de la cuerda, $p_{0}$ es la tensión inicial, $E$ es el módulo de Young del material, $\beta$ es el coeficiente de la fuerza amortiguadora, $g(t)$ es la función de relajación, y $f(u)$ es la fuerza restauradora. Cuando $\beta \equiv g \equiv 0$ y la cuerda es de material elástico, se tiene que la ecuación (1.7) es la propuesta y estudiada por Kirchhoff en [3]. El caso general $n \geq 1$ y $u=v$, la ecuación (1.1) tiene diversas aplicaciones, como en el área de la óptica no lineal, física del plasma, mecánica de fluidos y entre otras.

El caso $M \equiv 1, g_{i} \equiv 0$ y sin término disipativo, el sistema (1.1) - (1.2) describe la interacción de ciertas partículas elementales en un campo electromagnético, llamadas mesones. Un modelo matemático clásico que describe la interacción de los mesones es el sistema propuesto por Segal [14] que mostramos a continuación:

$$
\begin{aligned}
& u^{\prime \prime}-\Delta u+\alpha^{2} u+g^{2} v^{2} u=0, \\
& v^{\prime \prime}-\Delta v+\beta^{2} v+h^{2} u^{2} v=0,
\end{aligned}
$$

donde $\alpha$ y $\beta$ son las masas de los mesones $u$ y $v$ respectivamente y $g, h$ son las constantes de interacción.

Cuando $g_{i}$ son funciones no triviales, $M \equiv 1$ y sin considerar términos disipativos, el sistema (1.1) - (1.2) fue investigado por Andrade y Mognon [1], quienes obtienen existencia global para $f_{1}(u, v)=-|u|^{\rho-1} u|v|^{\rho}$ y $f_{2}(u, v)=-|v|^{\rho-1} v|u|^{\rho}$; por su parte Santos [13], obtiene existencia global y decaimiento exponencial para $f_{1}(u, v)=-\alpha(u-v)$ y $f_{2}(u, v)=\alpha(u-v)$. Cuando $g_{i} \equiv 0, M \equiv 1$ y sin considerar términos disipativos, el sistema (1.1) - (1.2) fue también estudiado por Milla Miranda y Medeiros [6], quienes obtienen existencia global para $f_{1}(u, v)=|v|^{\rho+2}|u|^{\rho} u-u$ y $f_{2}(u, v)=|u|^{\rho+2}|v|^{\rho} v-v$; asimismo, Li y Tsai [4], obtienen existencia local, existencia global y singularidad de soluciones para $f_{1}(u, v)=-m_{1}^{2} u-4 \lambda(u+\alpha v)^{3}-2 \beta u v^{2}$ y $f_{2}(u, v)=-m_{2}^{2} v-4 \alpha \lambda(u+\alpha v)^{3}-2 \beta u^{2} v$. Cuando $g_{i} \equiv 0$ y $M$ es una función no trivial, el sistema (1.1) - (1.2) fue estudiado por Quispe Méndez [7,9]: quien obtiene soluciones locales y singularidad de soluciones para $f_{i}$ específicas y sin términos disipativos; por su parte Wu y Tsai [18], obtienen existencia local y singularidad de soluciones para $f_{i}$ genéricas y adicionando términos disipativos. Cuando $g_{i}$ y $M$ son funciones no triviales, el sistema (1.1) - (1.2) fue estudiado por Quispe Méndez y Carrillo Díaz [12]; quienes obtienen soluciones locales para $f_{i}$ genéricas y con términos disipativos.

En este trabajo probaremos la propiedad de singularidad en tiempo finito de las soluciones del problema (1.1) - (1.6), cuando $g_{i}$ son funciones continuas no negativas, $M$ es una función continua positiva y las $f_{i}$ son funciones reales no lineales. Obtendremos la singularidad de soluciones, con energía inicial negativa, nula y positiva restringida, empleando el método directo [4]. Asimismo hallaremos las estimativas para el tiempo finito de explosión. En la discusión del problema emplearemos las estrategias y técnicas inspiradas en los trabajos de Wu y Tsai $[16,17]$ y Quispe Méndez [11].

\section{PRELIMINARES}

En esta sección presentamos algunas notaciones, conceptos y resultados sin demostración, los cuales serán usados en el desarrollo del presente trabajo.

Sea $\Omega$ un conjunto abierto y acotado de $\mathbb{R}^{n}$, con frontera suficientemente $\partial \Omega$. Denotamos el producto interno y la norma de $L^{2}(\Omega)$ y $L^{p}(\Omega)$, con $(.,$.$) y \mid$. $\left.\right|_{p}$. respectivamente, para $1 \leq p \leq \infty$. Además $((.,)$.$) y$ \|. If denotaran el producto interno y la norma de $H_{0}^{1}(\Omega)$, donde $((u, v)):=\int_{\Omega} \nabla u(x) . \nabla v(x) d x$ es la forma de Dirichlet.

Sea $X$ un espacio de Banach, $T$ y $p$ números reales tales que $0<T \leq \infty$ y $1 \leq p \leq \infty$. Representamos con $L^{p}(0, T ; X)$ al espacio de Banach de las funciones vectoriales $\left.u:\right] 0, T[\longrightarrow X$ medibles con $\|u(t)\|_{X} \in L^{p}(0, T)$, dotado de la norma

$$
\begin{aligned}
\|u\|_{L^{p}(0, T ; X)}:= & \left(\int_{0}^{T}\|u(t)\|_{X}^{p} d t\right)^{\frac{1}{p}}, 1 \leq p<\infty, \\
\|u\|_{L^{\infty}(0, T ; X)} & :=\sup _{0<t<T} \operatorname{ess}\|u(t)\|_{X}, p=\infty .
\end{aligned}
$$


Similarmente, cuando $0<T<\infty$, representamos con $C([0, T] ; X)$ al espacio de Banach de las funciones continuas $u:[0, T] \longrightarrow X$, dotado de la norma

$$
\|u\|_{C([0, T] ; X)}:=\sup _{0 \leq t \leq T}\|u(t)\|_{X} .
$$

Denotamos $w^{\prime}:=\frac{\partial w}{\partial t}, w^{\prime \prime}:=\frac{\partial^{2} w}{\partial t^{2}}, w(t)(x):=w(x, t), L^{1}:=L^{1}(0, T)$ y $L^{\infty}:=L^{\infty}(\Omega)$.

Hipótesis. Imponemos sobre las funciones reales $g_{i}(t), M(s)$ y $f_{i}(r, s)$ las siguientes condiciones:

(H1) $g_{i} \in C^{1}\left(\left[0, \infty[)\right.\right.$, acotada, $g_{i}(t) \geq 0, \forall t \geq 0$, existe una constante positiva $m_{0}$ tal que

$$
m_{0}-\int_{0}^{\infty} g_{i}(s) d s:=l_{i}>0
$$

para $i=1,2$, y existen constantes positivas $C_{1}$ y $C_{2}$ tales que

$$
-C_{1} g_{i}(t) \leq g_{i}^{\prime}(t) \leq-C_{2} g_{i}(t), \forall t \geq 0
$$

para $i=1,2$.

(H2) $M \in C^{1}\left(\left[0, \infty[)\right.\right.$ y $M(s) \geq m_{0}>0, \forall s \geq 0$, donde $m_{0}$ es la constante dada en (H1).

(H3) $f_{i} \in C^{1}\left(\mathbb{R}^{2}\right)$ para $i=1,2$, y para cada $(u, v) \in H_{0}^{1}(\Omega) \times H_{0}^{1}(\Omega)$, tenemos $u f_{1}(u, v)+v f_{2}(u, v) \in L^{1}(\Omega)$ y $F(u, v) \in L^{1}(\Omega)$, donde

$$
F(u, v):=\int_{0}^{u} f_{1}(\xi: v) d \xi+\int_{0}^{v} f_{2}(0, \xi) d \xi .
$$

(H4) $f_{i}(0,0)=0, i=1,2$ y existe una constante positiva $K$ tal que

$$
\begin{aligned}
\left|f_{i}\left(r_{1}, s_{1}\right)-f_{i}\left(r_{2}, s_{2}\right)\right| \leq & K\left[\left(\left|r_{1}\right|^{\alpha}+\left|r_{2}\right|^{\alpha}\right)\left|r_{1}-r_{2}\right|\right. \\
& \left.+\left(\left|s_{1}\right|^{\beta}+\left|s_{2}\right|^{\beta}\right)\left|s_{1}-s_{2}\right|\right],
\end{aligned}
$$

para cada $\left(r_{1}, s_{1}\right),\left(r_{2}, s_{2}\right) \in \mathbb{R}^{2}, i=1,2, \operatorname{con} 0 \leq \alpha, \beta \leq \frac{2}{n-2}$ para $n \geq 3$ ó $\alpha, \beta \geq 0$ para $n \leq 2$.

$(H 5) \frac{\partial f_{1}}{\partial s}(r, s)=\frac{\partial f_{2}}{\partial r}(r, s), \forall(r, s) \in \mathbb{R}^{2}$.

(H6) Existe una constante positiva $\gamma$ tal que

$$
r f_{1}(r, s)+s f_{2}(r, s) \geq 2(2 \gamma+1) F(r, s), \forall(r, s) \in \mathbb{R}^{2},
$$

donde $F(r, s)$ es la función dada en $(H 3)$.

(H7) $(2 \gamma+1) \widehat{M}(s) \geq\left(M(s)+2 \gamma m_{0}\right) s, \forall s \geq 0$, donde $\widehat{M}(s):=\int_{0}^{s} M(\xi) d \xi ; m_{0} \mathrm{y} \gamma$ son constantes dadas en $(H 1)$ y $(H 6)$ respectivamente.

(H8) $(4 \gamma+1) \int_{0}^{\infty} g_{i}(s) d s<4 \gamma m_{0}$ para $i=1,2$, donde $m_{0}$ y $\gamma$ son las constantes dadas en $(H 1)$ y $(H 6)$ respectivamente.

Lema 2.1 (Desigualdad de Sobolev-Poincaré [2]). Si $2 \leq p \leq \frac{2 n}{n-2}$ para $n \geq 3$ ó $p \geq 2$ para $n \leq 2$, entonces existe una constante positiva $B_{1}$ tal que

$$
|u|_{p} \leq B_{1}\|u\|, \forall u \in H_{0}^{1}(\Omega) .
$$

Lema 2.2. Si $g \in C^{1}\left(\left[0, \infty[)\right.\right.$ y $w \in C^{1}\left([0, T] ; L^{2}(\Omega)\right)$, entonces

$$
\begin{aligned}
\int_{0}^{t} g(t-s)\left(w(s), w^{\prime}(t)\right) d s= & -\frac{1}{2} \frac{d}{d t}\left[(g \square w)(t)-\left(\int_{0}^{t} g(s) d s\right)|w(t)|_{2}^{2}\right] \\
& -\frac{1}{2} g(t)|w(t)|_{2}^{2}+\frac{1}{2}\left(g^{\prime} \square w\right)(t),
\end{aligned}
$$


donde

$$
(v \square w)(t):=\int_{0}^{t} v(t-s)|w(t)-w(s)|_{2}^{2} d s .
$$

Demostración. Diferenciando el término $g \square w$, se obtiene el resultado.

Definición 2.3. Al par de funciones $(u, v)$ se le llama solución del problema (1.1) - (1.6) sobre [0,T], si las funciones $u, v: \Omega \times[0, T] \rightarrow \mathbb{R}$ satisfacen las condiciones (1.3) - (1.6) y verifican las igualdades siguientes:

$$
\begin{aligned}
& u^{\prime \prime}-M\left(|\nabla u|_{2}^{2}\right) \Delta u+g_{1} * \Delta u-\Delta u^{\prime}=f_{1}(u, v) \text { en } L^{2}\left(0, T ; H^{-1}(\Omega)\right), \\
& v^{\prime \prime}-M\left(|\nabla v|_{2}^{2}\right) \Delta v+g_{2} * \Delta v-\Delta v^{\prime}=f_{2}(u, v) \text { en } L^{2}\left(0, T ; H^{-1}(\Omega)\right) .
\end{aligned}
$$

Teorema 2.4 (Existencia Local [12]). Supongamos que las funciones $g_{i}, M$ y $f_{i}$ satisfacen las hipótesis (H1), (H2) y (H4) respectivamente, $u_{0}, v_{0} \in H_{0}^{1}(\Omega) \cap H^{2}(\Omega)$ y $u_{1}, v_{1} \in L^{2}(\Omega)$. Entonces existe un único intervalo $\left[0, T_{\text {máx }}\left[\right.\right.$ con $0<T_{\text {máx }} \leq \infty$ y el problema $(1.1)-(1.6)$ admite solución única $(u, v)$ sobre $\left[0, T_{\text {máx }}[\right.$ tal que

$$
\begin{aligned}
& u, v \in C\left(\left[0, T_{\text {máx }}\left[; H_{0}^{1}(\Omega) \cap H^{2}(\Omega)\right),\right.\right. \\
& u^{\prime}, v^{\prime} \in C\left(\left[0, T_{\text {máx }}\left[L^{2}(\Omega)\right) \cap L^{2}\left(0, T_{\text {máx }} ; H_{0}^{1}(\Omega)\right),\right.\right. \\
& u^{\prime \prime}, v^{\prime \prime} \in L^{2}\left(0, T_{\text {máx }} ; L^{2}(\Omega)\right) .
\end{aligned}
$$

Lema 2.5 ([4]). Sea $\gamma>0$ y sea $B \in C^{2}([0, \infty[)$ una función no negativa que satisface

$$
B^{\prime \prime}(t)-4(\gamma+1) B^{\prime}(t)+4(\gamma+1) B(t) \geq 0
$$

Si $B^{\prime}(0)>r_{2} B(0)+K_{0}$, entonces $B^{\prime}(t)>K_{0}$, para $t>0$, donde $K_{0}$ es una constante $y$

$$
r_{2}:=2(\gamma+1)-2 \sqrt{(\gamma+1) \gamma}
$$

es la menor raíz de la ecuación cuadrática $r^{2}-4(\gamma+1) r+4(\gamma+1)=0$.

Lema $2.6([4])$. Si $J(t)$ es una función no creciente en $\left[t_{0}, \infty\left[, t_{0} \geq 0\right.\right.$ y satisface la inecuación diferencial

$$
\left[J^{\prime}(t)\right]^{2} \geq a+b[J(t)]^{2+\frac{1}{\gamma}}, \text { para } t \geq t_{0},
$$

donde $a>0, \gamma>0$ y $b \in \mathbb{R}$, entonces existe un número real positivo $T_{*}$ tal que $\lim _{t \rightarrow T_{*}^{-}} J(t)=0$ y una cota superior de $T_{*}$ puede ser estimado respectivamente, en los siguientes casos:

(i) Si $b<0$ y $J\left(t_{0}\right)<\min \left\{1 \cdot \sqrt{\frac{a}{-b}}\right\}$. entonces

$$
T_{*} \leq t_{0}+\frac{1}{\sqrt{-b}} \ln \left(\frac{\sqrt{\frac{a}{-b}}}{\sqrt{\frac{a}{-b}}-J\left(t_{0}\right)}\right) .
$$

(ii) Si $b=0$, entonces

$$
T_{*} \leq t_{0}+\frac{J\left(t_{0}\right)}{\sqrt{a}} .
$$

(iii) Si $b>0$, entonces

$$
T_{*} \leq \frac{J\left(t_{0}\right)}{\sqrt{a}}
$$

$o$

$$
T_{*} \leq t_{0}+2^{\frac{3 \gamma+1}{2 \gamma}} \frac{\gamma c}{\sqrt{a}}\left(1-\left[1+c \cdot J\left(t_{0}\right)\right]^{-\frac{1}{2 \gamma}}\right):
$$

donde $c:=\left(\frac{b}{a}\right)^{\frac{1}{1+2}}$ 


\section{EL RESULTADO PRINCIPAL}

Ei objetivo principal del presente trabajo es discutir la propiedad de singularidad en tiempo finito de las soluciones del problema (1.1) - (1.6) sobre un intervalo maximal $\left[0, T_{\text {máx }}[\right.$. En la discusión usaremos el método directo [4].

Definición 3.1. Una solución $(u, v)$ del problema (1.1) - (1.6) sobre $\left[0, T_{\text {máx }}[\right.$ tiene la propiedad de explosión o singularidad en tiempo finito, si

$$
T_{\text {máx }}<\infty \mathrm{y} \operatorname{limm}_{t \rightarrow T_{\max }^{-}} \int_{\Omega}\left(|\nabla u(x, t)|^{2}+|\nabla v(x, t)|^{2}\right) d x=\infty .
$$

Definición 3.2. La función energía $E(t)$ del problema (1.1) - (1.6), se define por

$$
\begin{aligned}
E(t):=\frac{1}{2} & {\left[\left|u^{\prime}(t)\right|_{2}^{2}+\left|v^{\prime}(t)\right|_{2}^{2}\right]+\frac{1}{2}\left[\widehat{M}\left(\|u(t)\|^{2}\right)+\widehat{M}\left(\|v(t)\|^{2}\right)\right] } \\
& +\frac{1}{2}\left[\left(g_{1} \square \nabla u\right)(t)+\left(g_{2} \square \nabla v\right)(t)\right] \\
& -\frac{1}{2}\left[\left(\int_{0}^{t} g_{1}(s) d s\right)\|u(t)\|^{2}+\left(\int_{0}^{t} g_{1}(s) d s\right)\|v(t)\|^{2}\right] \\
& -\int_{\Omega} F(u(x, t), v(x, t)) d x,
\end{aligned}
$$

para $t \geq 0$, donde

$$
\begin{gathered}
\widehat{M}(s):=\int_{0}^{s} M(\xi) d \xi \\
F(r, s):=\int_{0}^{r} f_{1}(\xi, s) d \xi+\int_{0}^{s} f_{2}(0, \xi) d \xi
\end{gathered}
$$

y

$$
(g \square \nabla w)(t):=\int_{0}^{t} g(t-s)\|w(t)-w(s)\|^{2} d s .
$$

Lema 3.3. Supongamos que se cumplen las hipótesis (H1)-(H5). Si $(u, v)$ es una solución del problema (1.1)-(1.6) sobre $\left[0, T_{\max }\left[\right.\right.$ con datos iniciales $u_{0}, v_{0} \in H_{0}^{1}(\Omega) \cap H^{2}(\Omega)$ y $u_{1}, v_{1} \in L^{2}(\Omega)$, entonces

$$
\begin{aligned}
& E(t)+\int_{0}^{t}\left[\left\|u^{\prime}(s)\right\|^{2}+\left\|v^{\prime}(s)\right\|^{2}\right] d s \\
& +\frac{1}{2} \int_{0}^{t}\left[g_{1}(s)\|u(s)\|^{2}+g_{2}(s)\|v(s)\|^{2}\right] d s \\
& -\frac{1}{2} \int_{0}^{t}\left[\left(g_{1}^{\prime} \square \nabla u\right)(s)+\left(g_{2}^{\prime} \square \nabla v\right)(s)\right] d s=E(0),
\end{aligned}
$$

para $t \geq 0$, donde $E(0)$ es la energía inicial definida por

$$
\begin{aligned}
E(0):= & \frac{1}{2}\left[\left|u_{1}\right|_{2}^{2}+\left|v_{1}\right|_{2}^{2}\right]+\frac{1}{2}\left[\widehat{M}\left(\left\|u_{0}\right\|^{2}\right)+\widehat{M}\left(\left\|v_{0}\right\|^{2}\right)\right] \\
& -\int_{\Omega} F\left(u_{0}(x), v_{0}(x)\right) d x .
\end{aligned}
$$

Demostración. Multiplicando a la eculación (1.1) por $u^{\prime}$ y a la ecuación (1.2) por $v^{\prime}$. sumando estos resultados, integrando sobre $\Omega$, utilizando el teorema de la Divergencia, el Lema 2.2, (H3) y $(H 5)$, obtenemos

$$
\begin{aligned}
& E^{\prime}(t)+\left[\left\|u^{\prime}(t)\right\|^{2}+\left\|v^{\prime}(t)\right\|^{2}\right] \\
& +\frac{1}{2}\left[g_{1}(t)\|u(t)\|^{2}+g_{2}(t)\|v(t)\|^{2}\right] \\
& -\frac{1}{2}\left[\left(g_{1}^{\prime} \square \nabla u\right)(t)+\left(g_{2}^{\prime} \square \nabla v\right)(t)\right]=0 .
\end{aligned}
$$

De aquí, se obtiene el resultado.

Definición 3.4. Para una solución $(u, v)$ del problema $(1.1)$ - (1.6) sobre $\left[0, T_{\operatorname{máx}}[\right.$ : se define la función explosión

$$
A(t):=\left[|u(t)|_{2}^{2}+|v(t)|_{2}^{2}\right]+\int_{0}^{t}\left[\|u(s)\|^{2}+\|v(s)\|^{2}\right] d s, \text { para } t \geq 0 .
$$


Lema 3.5. Supongamos que se cumplen las hipótesis (H1)-(H8). Si $(u, v)$ es una solución del problema (1.1)-(1.6) sobre $\left[0, T_{\text {máx }}\left[\right.\right.$ con datos iniciales $u_{0}, v_{0} \in H_{0}^{1}(\Omega) \cap H^{2}(\Omega)$ y $u_{1}, v_{1} \in L^{2}(\Omega)$, entonces

$$
\begin{aligned}
A^{\prime \prime}(t) & -4(\gamma+1)\left[\left|u^{\prime}(t)\right|_{2}^{2}+\left|v^{\prime}(t)\right|_{2}^{2}\right. \\
& \left.+\int_{0}^{t}\left[\left\|u^{\prime}(s)\right\|^{2}+\left\|v^{\prime}(s)\right\|^{2}\right] d s\right] \geq-4(2 \gamma+1) E(0),
\end{aligned}
$$

para $t \geq 0$, donde $\gamma$ es la constante dada en $(H 6)$.

Demostración. Por diferenciación de (3.2), se tiene

$$
A^{\prime}(t)=2\left[\left(u^{\prime}(t), u(t)\right)+\left(v^{\prime}(t), v(t)\right)\right]+\left[\|u(t)\|^{2}+\|v(t)\|^{2}\right] .
$$

Diferenciando (3.4), utilizando las ecuaciones (1.1) - (1.2) y el teorema de la Divergencia, se obtiene

$$
\begin{aligned}
A^{\prime \prime}(t)= & 2\left[\left|u^{\prime}(t)\right|_{2}^{2}+\left|v^{\prime}(t)\right|_{2}^{2}\right] \\
& -2\left[M\left(\|u(t)\|^{2}\right)\|u(t)\|^{2}+M\left(\|v(t)\|^{2}\right)\|v(t)\|^{2}\right] \\
& +2\left[\left(f_{1}(u(t), v(t)), u(t)\right)+\left(f_{2}(u(t), v(t)), v(t)\right)\right] \\
& +2\left[\int_{0}^{t} g_{1}(t-s)((u(s), u(t))) d s\right. \\
& \left.+\int_{0}^{t} g_{2}(t-s)((v(s), v(t))) d s\right] .3 .5
\end{aligned}
$$

De (3.5) y (3.1), resulta

$$
\begin{aligned}
& A^{\prime \prime}(t)-4(\gamma+1)\left[\left|u^{\prime}(t)\right|_{2}^{2}+\left|v^{\prime}(t)\right|_{2}^{2}\right. \\
& \left.+\int_{0}^{t}\left[\left\|u^{\prime}(s)\right\|^{2}+\left\|v^{\prime}(s)\right\|^{2}\right] d s\right]=-4(2 \gamma+1) E(0) \\
& \quad+2 \int_{\Omega}\left[u f_{1}(u, v)+v f_{2}(u, v)-2(2 \gamma+1) F(u, v)\right] d x \\
& \quad+2(2 \gamma+1)\left[\widehat{M}\left(\|u(t)\|^{2}\right)+\widehat{M}\left(\|v(t)\|^{2}\right)\right] \\
& \quad-2\left[M\left(\|u(t)\|^{2}\right)+(2 \gamma+1) \int_{0}^{t} g_{1}(s) d s\right]\|u(t)\|^{2} \\
& \quad-2\left[M\left(\|v(t)\|^{2}\right)+(2 \gamma+1) \int_{0}^{t} g_{2}(s) d s\right]\|v(t)\|^{2} \\
& \quad+2\left[\int_{0}^{t} g_{1}(t-s)((u(s), u(t))) d s+\int_{0}^{t} g_{2}(t-s)((v(s), v(t))) d s\right] \\
& \quad-2(2 \gamma+1)\left[\int_{0}^{t}\left(g_{1}^{\prime} \square \nabla u\right)(s) d s+\int_{0}^{t}\left(g_{2}^{\prime} \square \nabla v\right)(s) d s\right] \\
& \quad+2(2 \gamma+1)\left[\int_{0}^{t} g_{1}(s)\|u(s)\|^{2} d s+\int_{0}^{t} g_{2}(s)\|v(s)\|^{2} d s\right] \\
& \quad+2(2 \gamma+1)\left[\left(g_{1} \square \nabla u\right)(t)+\left(g_{2} \square \nabla v\right)(t)\right] \\
& \quad+4 \gamma\left[\int_{0}^{t}\left\|u^{\prime}(s)\right\|^{2} d s+\int_{0}^{t}\left\|v^{\prime}(s)\right\|^{2} d s\right] .
\end{aligned}
$$

Utilizando la desigualdad $2\left(\left(w_{1}, w_{2}\right)\right) \geq-\left[\left\|w_{1}\right\|^{2}+\left\|w_{2}\right\|^{2}\right]$. resulta

$$
\begin{aligned}
2 \int_{0}^{t} g(t-s)((w(s), w(t))) d s= & 2 \int_{0}^{t} g(t \cdots s)((w \cdot(s)-w(t), w(t))) d s \\
& +2\left(\int_{0}^{t} g(s) d s\right)\|w(t)\|^{2} \\
\geq & -\left[(g \square \nabla w)(t)+\left(\int_{0}^{t} g(s) d s\right)\|w(t)\|^{2}\right] \\
& +2\left(\int_{0}^{t} g(s) d s\right)\|w(t)\|^{2} \\
= & \left(\int_{0}^{t} g(s) d s\right)\|w(t)\|^{2}-(g \square \nabla w)(t) .3 .7
\end{aligned}
$$

Por las hipótesis $(H 1)$, (H6) - (H8) y utilizando (3.7), se obtiene de (3.6) el resultado (3.3).

Lema 3.6. Supongamos que se cumplen las hipótesis $(H 1)-(H 8)$. Si $(u, v)$ es una solución del problema (1.1)-(1.6) sobre $\left[0 . T_{\text {máx }}\right.$ con datos iniciales $u_{0}, v_{0} \in H_{0}^{1}(\Omega) \cap H^{2}(\Omega)$ y $u_{1}, v_{1} \in L^{2}(\Omega)$, y satisfaciendo una de las siguientes condiciones: 
(i) $E(0)<0$,

(ii) $E(0)=0 \quad$ y $A^{\prime}(0)>K_{0}$,

(iii) $E(0)>0 \quad$ y $\quad A^{\prime}(0)>r_{2}\left[A(0)+\frac{K_{1}}{4(\gamma+1)}\right]+K_{0}$,

donde

$$
\begin{gathered}
K_{0}:=\left\|u_{0}\right\|^{2}+\left\|v_{0}\right\|^{2}, \\
A(0):=\left|u_{0}\right|_{2}^{2}+\left|v_{0}\right|_{2}^{2}, \quad A^{\prime}(0):=2\left[\left(u_{1}, u_{0}\right)+\left(v_{1}, v_{0}\right)\right]+K_{0}, \\
K_{1}:=4(2 \gamma+1) E(0)+4(\gamma+1) K_{0}, \\
r_{2}:=2(\gamma+1)-2 \sqrt{(\gamma+1) \gamma},
\end{gathered}
$$

entonces

$$
A^{\prime}(t)>K_{0}, \text { para } t>t_{0},
$$

donde $t_{0}:=\operatorname{máx}\left\{\frac{A^{\prime}(0)-K_{0}}{4(1+2 \gamma) E(0)}, 0\right\}$ en el caso (i) y $t_{0}:=0$ en los casos (ii) y (iii).

Demostración. Consideremos tres casos de acuerdo al signo de la energía inicial $E(0)$.

(i.) Si $E(0)<0$, de (3.3), se tiene

$$
A^{\prime \prime}(t) \geq-4(2 \gamma+1) E(0)
$$

e integrando, resulta

$$
A^{\prime}(t) \geq A^{\prime}(0)-4(2 \gamma+1) E(0) t, \text { para } t \geq 0 .
$$

Considerando $A^{\prime}(0)-K_{0}-4(2 \gamma+1) E(0) t>0$, se obtiene

$$
A^{\prime}(t)>K_{0}, \text { para } t>t_{0},
$$

donde

$$
t_{0}:=\max \left\{\frac{A^{\prime}(0)-K_{0}}{4(1+2 \eta) E(0)}, 0\right\} .
$$

(ii) Si $E(0)=0$, de (3.3), se tiene

$$
A^{\prime \prime}(t) \geq 0
$$

e integrando. resulta

$$
A^{\prime}(t) \geq A^{\prime}(0), \text { para } t \geq 0 .
$$

Considerando $A^{\prime}(0)-K_{0}>0$, se obtiene

$$
A^{\prime}(t)>K_{0}, \text { para } t \geq 0 .
$$

(iii) Para $E(0)>0$. Primero notemos que se cumple

$$
2 \int_{0}^{t}\left(\left(w^{\prime}(s) \cdot w(s)\right)\right) d s=\|w(t)\|^{2}-\left\|w_{0}\right\|^{2} .
$$

Usando la desigualdad de Hölder en (3.9), se obtiene

$$
\|w(t)\|^{2} \leq\left\|w_{0}\right\|^{2}+\int_{0}^{t}\|w \cdot(s)\|^{2} d s+\int_{0}^{t}\left\|w^{\prime}(s)\right\|^{2} d s .
$$

Nuevamente usando la desigualdad de Hölder en (3.4) y por (3.10). resulta

$$
\begin{aligned}
A^{\prime}(t) \leq & A(t)+K_{0}+\left[\left|u^{\prime}(t)\right|_{2}^{2}+\left|v^{\prime}(t)\right|_{2}^{2}\right] \\
& +\int_{0}^{t}\left[\left\|u^{\prime}(s)\right\|_{2}^{2}+\left\|v^{\prime}(s)\right\|_{2}^{2}\right] d s .3 .11
\end{aligned}
$$


De (3.3) y (3.11), obtenemos

$$
A^{\prime \prime}(t)-4(\gamma+1) A^{\prime}(t)+4(\gamma+1) A(t)+K_{1} \geq 0
$$

donde

$$
K_{1}:=4(2 \gamma+1) E(0)+4(\gamma+1) K_{0}
$$

Definamos la función

$$
B(t):=A(t)+\frac{K_{1}}{4(\gamma+1)}, \text { para } t \geq 0
$$

Considerando $B^{\prime}(0)>r_{2} B(0)+K_{0}$, la función $B$ satisface las condiciones del Lema 2.5. Así se tiene $A^{\prime}(t)>K_{0}$, para $t>0$. Con esto se concluye la prueba del Lema 3.6.

Definición 3.7. Para las estimativas del tiempo finito de la función explosión $A$, definamos la función

$$
J(t):=\left[A(t)+\left(T_{1}-t\right) K_{0}\right]^{-\gamma}, \text { para } t \in\left[0, T_{1}\right],
$$

donde $T_{1}$ es uma constante positiva que será determinada posteriormente y $\gamma$ es la constante dada en (H6).

Teorema 3.8 (Singularidad de Soluciones). Supongamos que se cumplen las hipótesis $(H 1)-(H 8)$. Si $(u, v)$ es una solución del problema (1.1) - (1.6) sobre $\left[0, T_{\text {máx }}\right.$ [ con datos iniciales $u_{0}, v_{0} \in H_{0}^{1}(\Omega) \cap H^{2}(\Omega)$ y $u_{1}, v_{1} \in L^{2}(\Omega)$, y satisfaciendo una de las siguientes condiciones:

(i) $E(0)<0$,

(ii) $E(0)=0 \quad$ y $A^{\prime}(0)>K_{0}$,

(iii) $0<E(0)<\frac{\left[A^{\prime}(0)-K_{0}\right]^{2}}{8\left[A(0)+T_{1} K_{0}\right]}$ y $A^{\prime}(0)>r_{2}\left[A(0)+\frac{K_{1}}{4(\gamma+1)}\right]+K_{0}$ ：

entonces $T_{\text {máx }}<\infty y \operatorname{lím}_{t \rightarrow T_{\operatorname{mix}}^{-}}\left[\|u(t)\|^{2}+\|v(t)\|^{2}\right]=\propto$. Adcmás el tiempo finito $T_{\text {máx }}$ es estimado, en el caso (i)

$$
T_{\text {máx }} \leq t_{0}-\frac{J\left(t_{0}\right)}{J^{\prime}\left(t_{0}\right)}
$$

Además, si . $J\left(t_{0}\right)<\operatorname{mín}\left\{1, \sqrt{\frac{a}{-b}}\right\}$, entonces

$$
T_{\text {máx }} \leq t_{0}+\frac{1}{\sqrt{-b}} \ln \left(\frac{\sqrt{\frac{a}{-b}}}{\sqrt{\frac{a}{-b}}-J\left(t_{0}\right)}\right) .
$$

En el caso (ii),

$$
T_{\text {máx }} \leq t_{0}-\frac{J\left(t_{0}\right)}{J^{\prime}\left(t_{0}\right)}
$$

o

$$
T_{\text {máx }} \leq t_{0}+\frac{J\left(t_{0}\right)}{\sqrt{a}}
$$

En el caso (iii),

$$
T_{\text {máx }} \leq \frac{J\left(t_{0}\right)}{\sqrt{a}}
$$

o

$$
T_{\text {máx }} \leq t_{0}+2^{\frac{3 \gamma+1}{2 \gamma}} \frac{\gamma c}{\sqrt{a}}\left\{1-\left[1+c J\left(t_{0}\right)\right]^{-\frac{1}{2 \gamma}}\right\}
$$

donde $a:=\gamma^{2}\left[J\left(t_{0}\right)\right]^{\frac{2}{\gamma}+2}\left[\left[A^{\prime}\left(t_{0}\right)-K_{0}\right]^{2}-8 E(0)\left[J\left(t_{0}\right)\right]^{\frac{-1}{\gamma}}\right] \cdot b:=8 \gamma^{2} E(0)$ y $c:=\left(\frac{b}{a}\right)^{\frac{\gamma}{\gamma+2}}$.

En el caso $(i), t_{0}:=\operatorname{máx}\left\{\frac{A^{\prime}(0)-K_{0}}{4(1+2 \gamma) E(0)}, 0\right\}$ y $t_{0}:=0$ en los casos (ii) y (iii). 
Demostración. Por diferenciación de (3.12), resulta

$$
J^{\prime}(t)=-\gamma[J(t)]^{\frac{1}{\gamma}+1}\left[A^{\prime}(t)-K_{0}\right]
$$

y

$$
J^{\prime \prime}(t)=-\gamma[J(t)]^{\frac{2}{\gamma}+1} V(t)
$$

donde

$$
V(t):=A^{\prime \prime}(t)\left[A(t)+\left(T_{1}-t\right) K_{0}\right]-(\gamma+1)\left[A^{\prime}(t)-K_{0}\right]^{2} .
$$

Utilizando la desigualdad $\left(\sum_{i=1}^{4} a_{i} b_{i}\right)^{2} \leq\left(\sum_{i=1}^{4} a_{i}^{2}\right)\left(\sum_{i=1}^{4} b_{i}^{2}\right),(3.9)$ y la desigualdad de Hölder, de (3.4), resulta

$$
\begin{aligned}
{\left[A^{\prime}(t)-K_{0}\right]^{2} \leq } & 4\left[A(t)+\left(T_{1}-t\right) K_{0}\right]\left[\left|u^{\prime}(t)\right|_{2}^{2}+\left|v^{\prime}(t)\right|_{2}^{2}\right. \\
& \left.+\int_{0}^{t}\left[\left\|u^{\prime}(s)\right\|_{2}^{2}+\left\|v^{\prime}(s)\right\|_{2}^{2}\right] d s\right] .3 .21
\end{aligned}
$$

De (3.20) y (3.21), se tiene

$$
J^{\prime \prime}(t) \leq-\gamma[J(t)]^{\frac{1}{\gamma}+1} K(t),
$$

donde

$$
\begin{aligned}
K(t):=A^{\prime \prime}(t)-4(\gamma+1)\left[\left|u^{\prime}(t)\right|_{2}^{2}+\left|v^{\prime}(t)\right|_{2}^{2}\right. \\
\left.\quad+\int_{0}^{t}\left[\left\|u^{\prime}(s)\right\|^{2}+\left\|v^{\prime}(s)\right\|_{2}^{2}\right] d s\right] .
\end{aligned}
$$

Por (3.3) y (3.22), resulta

$$
J^{\prime \prime}(t) \leq 4 \gamma(2 \gamma+1) E(0)[J(t)]^{\frac{1}{\gamma}+1}, \text { para } t \geq t_{0} .
$$

De (3.8) y (3.19), se tiene

$$
J^{\prime}(t)<0 \text {, para } t>t_{0} .
$$

Multiplicando (3.23) por $J^{\prime}(t)$ y luego integrando de $t_{0}$ a $t$, se obtiene

$$
\left[J^{\prime}(t)\right]^{2} \geq a+b[J(t)]^{2+\frac{1}{2}}, \text { para } t \geq t_{0},
$$

donde

$$
\begin{aligned}
a & :=\left[J^{\prime}\left(t_{0}\right)\right]^{2}-8 \gamma^{2} E(0)\left[J\left(t_{0}\right)\right]^{\frac{1}{1}+2} \\
& =\gamma^{2}\left[J\left(t_{0}\right)\right]^{\frac{2}{\gamma}+2}\left[\left[A^{\prime}\left(t_{0}\right)-K_{0}\right]^{2}-8 E(0)\left[J\left(t_{0}\right)\right]^{\frac{-1}{3}}\right]
\end{aligned}
$$

y

$$
b:=8 \gamma^{2} E(0) \text {. }
$$

Observemos que $a>0$ si y solo si $E(0)<\frac{\left[A^{\prime}\left(t_{0}\right)-K_{0}\right]^{2}}{8\left[A\left(t_{0}\right)+\left(T_{1}-t_{0}\right) K_{0}\right]}$.

El caso particular en el que $E(0)<0$, por $(3.23)$ y $(3.24)$, se obticne directamente $\lim _{t \rightarrow T_{-}} J(t)=0$ y la estimativa (3.13) para el tiempo finito $T_{*}$. Para los demás casos, por (3.24) y (3.25). la función $J$ satisface las condiciones del Lema 2.6. Entonces existe un tiempo finito $T_{*}$ tal que $\lim _{t \rightarrow T_{\star}^{-}} J(t)=0$ y la cota superior para $T_{*}$ son estimadas respectivamente de acuerdo al signo de la energía inicial $E(0)$.

Observemos que las estimativas (3.15) y (3.16) son equivalentes, es decir $\sqrt{a}=-J^{\prime}\left(t_{0}\right)$.

Desde que [0. $T_{\text {máx }}$ [ es el intervalo maximal de las soluciones del problema (1.1) - (1.6). resulta que $T_{\text {máx }}=T_{*}$. También por $\lim _{t \rightarrow T_{\text {mix }}^{-}} J(t)=0$, se obtiene

$$
\lim _{t \rightarrow T_{\text {tuix }}^{-}} A(t)=\infty
$$


De aquí y la desigualdad de Sobolev-Poincaré, se deduce

$$
\lim _{t \rightarrow T_{\text {mix }}^{-}}\left[\|u(t)\|^{2}+\|v(t)\|^{2}\right]=\infty .
$$

Con todo esto se concluye la demostración del Teorema 3.8.

Observación 3.9. La selección de $T_{1}$ de (3.12) es posible escoger con algunas consideraciones. Veamos tres casos de acuerdo al signo de la energía inicial $E(0)$.

(a) Para el caso $E(0)=0$.

Por la condición $A^{\prime}(0)>K_{0}$, resulta $\left(u_{1}, u_{0}\right)+\left(v_{1}, v_{0}\right)>0$ y $t_{0}=0$. Por (3.15), escogemos

$$
T_{1} \geq \frac{A(0)+T_{1} K_{0}}{\gamma\left[A^{\prime}(0)-K_{0}\right]}=-\frac{J(0)}{J^{\prime}(0)}
$$

Aplicando la desigualdad de Hölder, desigualdad de Sobolev-Poincaré y la desigualdad de Young, resulta

$$
A(0)+T_{1} K_{0} \leq \gamma B_{1} T_{1}\left(\varepsilon K_{0}+\frac{1}{\varepsilon}\left[\left|u_{1}\right|_{2}^{2}+\left|v_{1}\right|_{2}^{2}\right]\right),
$$

donde $B_{1}$ es la constante de la desigualdad de Sobolev-Poincaré y $\varepsilon$ es cualquier constante positiva. Escogiendo $\varepsilon=\frac{1}{\gamma B_{1}}$, obtenemos

$$
T_{1} \geq \frac{A(0)}{\gamma^{2} B_{1}^{2}\left[\left|u_{1}\right|_{2}^{2}+\left|v_{1}\right|_{2}^{2}\right]} .
$$

En particular, podemos tomar

$$
T_{1}=\frac{A(0)}{\gamma^{2} B_{1}^{2}\left[\left|u_{1}\right|_{2}^{2}+\left|v_{1}\right|_{2}^{2}\right]} .
$$

(b) Para el caso $E(0)<0$.

Veamos dos casos de acuerdo al signo de $A^{\prime}(0)-K_{0}$.

$\left.b_{1}\right)$ Si $A^{\prime}(0)>K_{0}$, entonces $\left(u_{1}, u_{0}\right)+\left(v_{1}, v_{0}\right)>0$ y $t_{0}=0$. Por (3.13), podemos escoger $T_{1}$ como en el caso $(a)$.

$\left.b_{2}\right)$ Si $A^{\prime}(0)<K_{0}$. entonces $\left(u_{1}, u_{0}\right)+\left(v_{1}, v_{0}\right)<0$ y $t_{0}=\frac{\left(u_{1}, u_{0}\right)+\left(v_{1}, v_{0}\right)}{2(1+2 \gamma) E(0)}$. Por (3.13). teniendo en cuenta el signo de $A^{\prime}\left(t_{0}\right)-K_{0}$, escogemos $T_{1}$ que resuelva la inecuación

$$
T_{1} \geq t_{0}+\frac{A\left(t_{0}\right)+\left(T_{1}-t_{0}\right) K_{0}}{\gamma\left[A^{\prime}\left(t_{0}\right)-K_{0}\right]} .
$$

(c) Para el caso $E(0)>0$.

Definamos las siguientes constantes positivas:

$$
\begin{gathered}
\kappa_{1}:=\frac{(\gamma+1)\left[A^{\prime}(0)-r_{2} A(0)-\left(r_{2}+1\right) K_{0}\right]}{r_{2}(2 \gamma+1)}, \\
\kappa_{2}:=\frac{\left[A^{\prime}(0)-K_{0}\right]^{2}\left[c \gamma-K_{0}\right]}{8 c \gamma A(0)}, \\
\kappa_{3}:=\frac{A(0)}{c_{\gamma}-K_{0}}
\end{gathered}
$$

y

$$
\kappa_{4}:=\frac{\left[A^{\prime}(0)-K_{0}\right]^{2}-8 E(0) A(0)}{8 E(0) K_{0}}
$$

donde

$$
c:= \begin{cases}1, & \text { si } \gamma>K_{0} \\ \frac{2 K_{0}}{\gamma}, & \text { si } \gamma \leq K_{0}\end{cases}
$$


Entonces, resultan las siguientes equivalencias:

$$
A^{\prime}(0)>r_{2}\left[A(0)+\frac{K_{1}}{4(\gamma+1)}\right]+K_{0} \Longleftrightarrow E(0)<\kappa_{1}
$$

$\mathrm{y}$

$$
\kappa_{3} \leq \kappa_{4} \Longleftrightarrow E(0) \leq \kappa_{2} .
$$

Ahora hallemos la constante positiva $T_{1}$. Primero observemos las siguientes equivalencias con $T_{1}$ :

$$
\begin{gathered}
E(0)<\frac{\left[A^{\prime}(0)-K_{0}\right]^{2}}{8\left[A(0)+T_{1} K_{0}\right]} \Longleftrightarrow T_{1}<\kappa_{4}, \\
\frac{A(0)+T_{1} K_{0}}{c \gamma} \leq T_{1} \Longleftrightarrow \kappa_{3} \leq T_{1}
\end{gathered}
$$

$\mathrm{y}$

$$
\frac{c}{\sqrt{\left[A^{\prime}(0)-K_{0}\right]^{2}-8 E(0)\left[A(0)+T_{1} K_{0}\right]}}<1 \Longleftrightarrow T_{1}<\kappa_{4}-\frac{c^{2}}{8 E(0) K_{0}} .
$$

Por (3.17), debemos escoger $T_{1}$ que resuelva la inecuación

$$
\frac{A(0)+T_{1} K_{0}}{\gamma \sqrt{\left[A^{\prime}(0)-K_{0}\right]^{2}-8 E(0)\left[A(0)+T_{1} K_{0}\right]}} \leq \frac{A(0)+T_{1} K_{0}}{c \gamma} \leq T_{1} .
$$

Por (3.26) y (3.27), tenemos

$$
E(0)<\min \left\{\kappa_{1}, \kappa_{2}\right\}
$$

Por (3.32) y (3.28) - (3.30), el $T_{1}$ que satisface (3.31), debemos de escoger en el intervalo

$$
\kappa_{3} \leq T_{1} \leq \kappa_{4} .
$$

En particular, tomando $T_{1}=\kappa_{3}$, resulta

$$
T_{\text {máx }} \leq \frac{J\left(t_{0}\right)}{\sqrt{a}}=\frac{c \kappa_{3}}{\sqrt{\left[A^{\prime}(0)-K_{0}\right]^{2}-8 E(0) c \gamma \kappa_{3}}} .
$$

Con esto se concluye las posibles selecciones de $T_{1}$. 


\section{Bibliografía}

[1] Andrade D. and Mognon A. (2003), Global solutions for a system of Klein-Gordon equations with memory, Bol. Soc. Paran. Mat. 21 1/2.

[2] Brézis H. (1984). Análisis funcional, Alianza Editorial, Madrid.

[3] Kirchhoff G. (1883), Vorlesungen über mechanik, Leipzig, Teubner.

[4] Li, M.-R. and Tsai L.-Y. (2003), On a system of nonlinear wave equation, Taiwanese Journal of Mathematic Vol.7, No. 4, pp. 557-573.

[5] Messaoudi S. (2006): Blow-up of positive-initial-energy solutions of a nonlinear viscoelastic hyperbolic equation, J. Math. Anal. Appl. 320, 902-915.

[6] Milla Miranda, M. and Medeiros, L. A. (1987), On the existence of global solutions of a coupled nonlinear Klein-Gordon equation, Funkcialaj Ekvacioj, 30, 147-161.

[7] Quispe Méndez, T. (1998), Singularidad en tiempo finito para un sistema de Kirchhoff; Tesis de Maestria, Fac. CC. MM. de la UNMSM.

[8] Quispe Méndez, T. (2004). Singularidad de soluciones de una ecuación de Kirchhoff no lineal con término disipativo, PESQUIMAT Revista de la Fac. CC. MM. de la Universidad Nacional Mayor de San Marcos Vol. VII. No.1, pág. 18-29, LIMA-PERÚ.

[9] (Quispe Méndez, T. (2005). Solución local y singularidad para un sistema de Kirchhoff no lineal. PESQUIMAT Revista de la Fac. CC. MM. de la Universidad Nacional Mayor de San Marcos Vol. VIII, No.2, pp 45-62, LIMA-PERÚ.

[10] Quispe Méndez. T. (2007), Solución local de una ecuación de Kirchhoff no lineal viscoelástica con término disipativo, PESQUIMAT Revista de la Fac. CC. MM. de la Universidad Nacional Mayor de San Marcos Vol. X, No.1, pp 11-32, LIMA-PERÚ.

[11] Quispe Méndez, T. (2007). Singularidad de soluciones para una ecuación de Kirchhoff no lineal viscoelástica con término disipativo. PESQUIMAT Revista de la Fac. CC. MM. de la Universidad Nacional Mayor do San Marcos Vol. X. No.2. pp 67-צ(). LIMA-PERÚ.

[12] Quispe Méndez. T. y Carrillo Díaz, L. E. (2010). Solución local de un sistema de Kirchhoff no lineal viscoelástico con término disipativo. PESQUIMAT Revista de la Fac. CC. MIM. de la Universidad Nacional Mayor de San Marcos Vol. XIII, No.2. pp 40-58. LIMA-PERÚ.

[13] Santos, M. L. (2002). Decay rates for solutions of a system of wave cquations with memory: Electronic Journal of Differential Equations, Vol. 2002. No. 38. pp.1-17.

[14] Segal. I. (1965), Nonlinear partial differential equations in quantum fiels theory. Proc: Symp. Appl. Math. A.M.S.. 17. 210-226.

[15] Wu S.-T. (2006). Blow-up of solutions for an integro-differential equation with a nonlinear source, Electronic Journal of Differential Equations. Vol. 2006. No. 45, pp.1-9.

[16] Wu S.-T. and Tsai L.-Y. (2006), On global existence and blow-up of solutions for an integro-differential equation with strong damping. Taiwanese Journal of Mathematic Vol.10. No. 4. pp. 979-1014.

[17] Wu S.-T. and Tsai L.-Y. (2007): On system of nonlinear wave equation of Kirchhoff type with a strong dissipation, Tamkang Journal of Mathematic Vol.38. No. 1, pp. 1.20.

[18] Wil S.-T. (2009), Blou-up of solutions for a system of nonlinear wave equations with nonlinear damping: Electronic Journal of Differential Equations, Vol. 2009. No. 105. pp.1-11. 